\title{
Jejunal Intussusception Caused by a Nasointestinal Ileus Tube
}

\author{
Junpei Komagamine, Daichi Noritomi \\ Department of Internal Medicine, National Hospital Organization Tochigi Medical Center, Nakatomatsuri, Utsunomiya, Tochigi, Japan
}

Received: 02/01/2022

Accepted: $19 / 01 / 2022$

Published: 09/02/2022

How to cite this article: Komagamine J, Noritomi D. Jejunal intussusception caused by a nasointestinal ileus tube. EJCRIM 2022;9:doi:10.12890/2022_003161.

Conflicts of Interests: The Authors declare that there are no competing interests.

This article is licensed under a Commons Attribution Non-Commercial 4.0 License

\begin{abstract}
A 79-year-old man was admitted to our hospital due to pleural empyema. After 4 weeks of antimicrobial therapy and pleural drainage, he recovered but complained of new-onset abdominal pain. Abdominal computed tomography revealed adhesive small bowel obstruction and a nasointestinal ileus tube with intermittent suction was inserted. This procedure initially decreased his abdominal pain, but severe abdominal pain and vomiting developed 3 days later. Repeat abdominal computed tomography revealed jejuno-jejunal intussusception due to the nasointestinal ileus tube. Our patient was initially treated conservatively. However, he underwent surgical reduction due to clinical deterioration 1 day after diagnosis and died from a surgical complication 19 days later. Intussusception is a rare but fatal complication caused by placement of a nasointestinal ileus tube in the small intestine. Because urgent operative reduction is needed to avoid intestinal resection in most cases, early diagnosis and surgical reduction of intussusception are critical.
\end{abstract}

\section{KEYWORDS}

Intussusception, nasointestinal ileus tube

\section{LEARNING POINTS}

- Intussusception is a rare but fatal complication caused by placement of a nasointestinal ileus tube.

- Intussusception should be suspected if abdominal pain and distention worsen after placement of a nasointestinal ileus tube.

- Urgent operative reduction is needed for intussusception due to a nasointestinal ileus tube.

\section{CASE DESCRIPTION}

A 79-year-old man with schizophrenia and a history of appendectomy was admitted to our hospital due to pleural empyema. After 4 weeks of antimicrobial therapy and pleural drainage, he recovered but complained of new-onset abdominal pain. Abdominal computed tomography revealed adhesive small bowel obstruction. A nasointestinal ileus tube with intermittent suction was inserted, and intravenous hydration was started. These procedures initially decreased his abdominal pain, but severe abdominal pain and vomiting developed 3 days later. He was alert and oriented. His temperature was $36.2^{\circ} \mathrm{C}$, blood pressure $117 / 91 \mathrm{mmHg}$, pulse 95 beats per minute, respiratory rate 26 per minute, and oxygen saturation $96 \%$. On examination, an abdominal mass was palpated in the left upper quadrant, but no tenderness or signs of peritonitis were found.

Laboratory tests revealed an elevated lactate level of $2.3 \mathrm{mmol} / \mathrm{l}$ and an elevated C-reactive protein level of $5.2 \mathrm{mg} / \mathrm{dl}$. Repeat abdominal computed tomography revealed a typical target sign around the nasointestinal ileus tube (Fig. 1). Jejuno-jejunal intussusception due to a nasointestinal ileus tube was diagnosed. Our patient was initially treated conservatively. However, he underwent surgical reduction due to clinical deterioration 1 day after diagnosis, and the involved necrotic jejunal segment was resected (Fig. 2). Nonetheless, he died from a surgical complication 19 days later. 

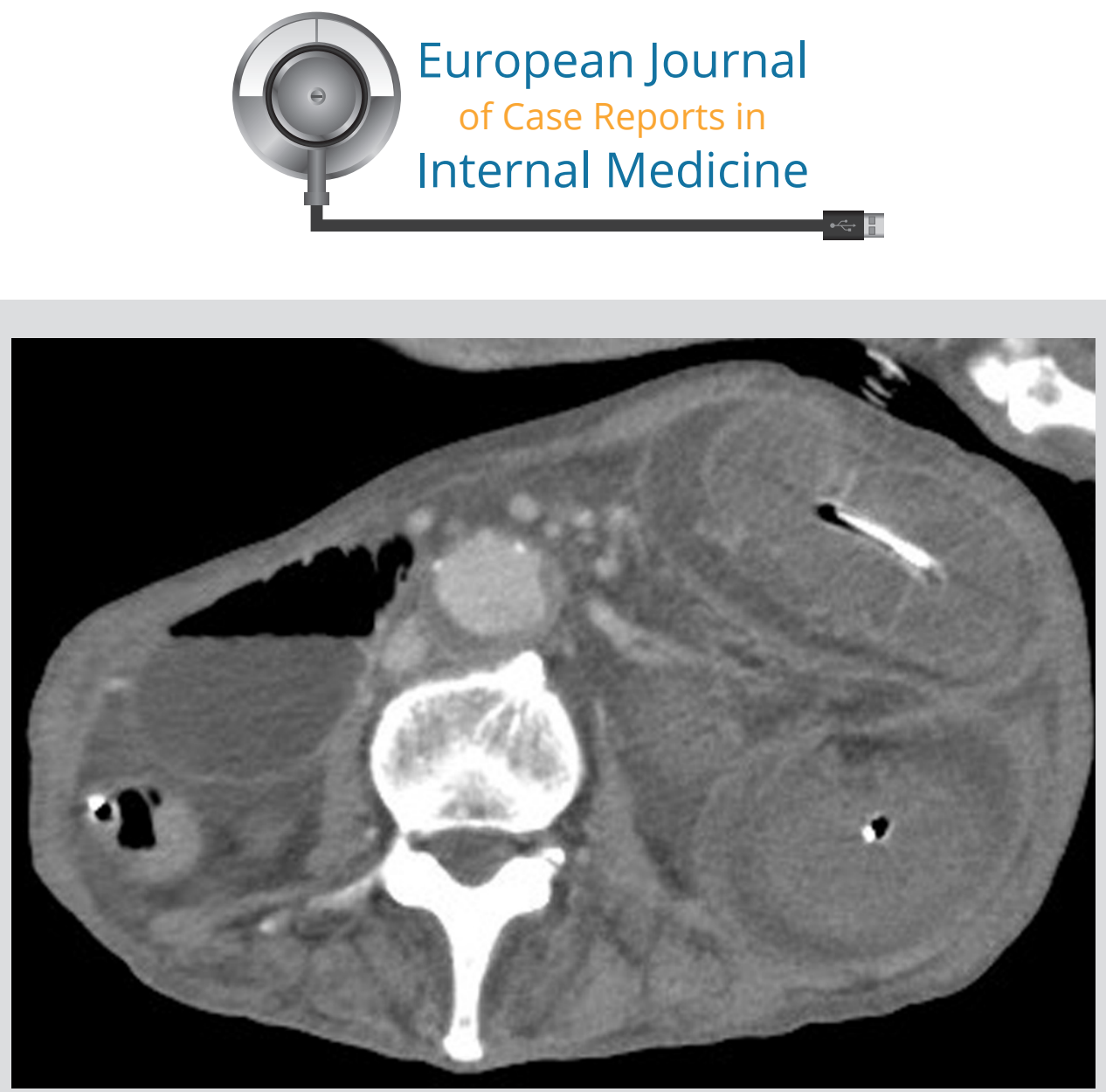

Figure 1. Abdominal contrast-enhanced computed tomography showed a typical target sign around the nasointestinal ileus tube

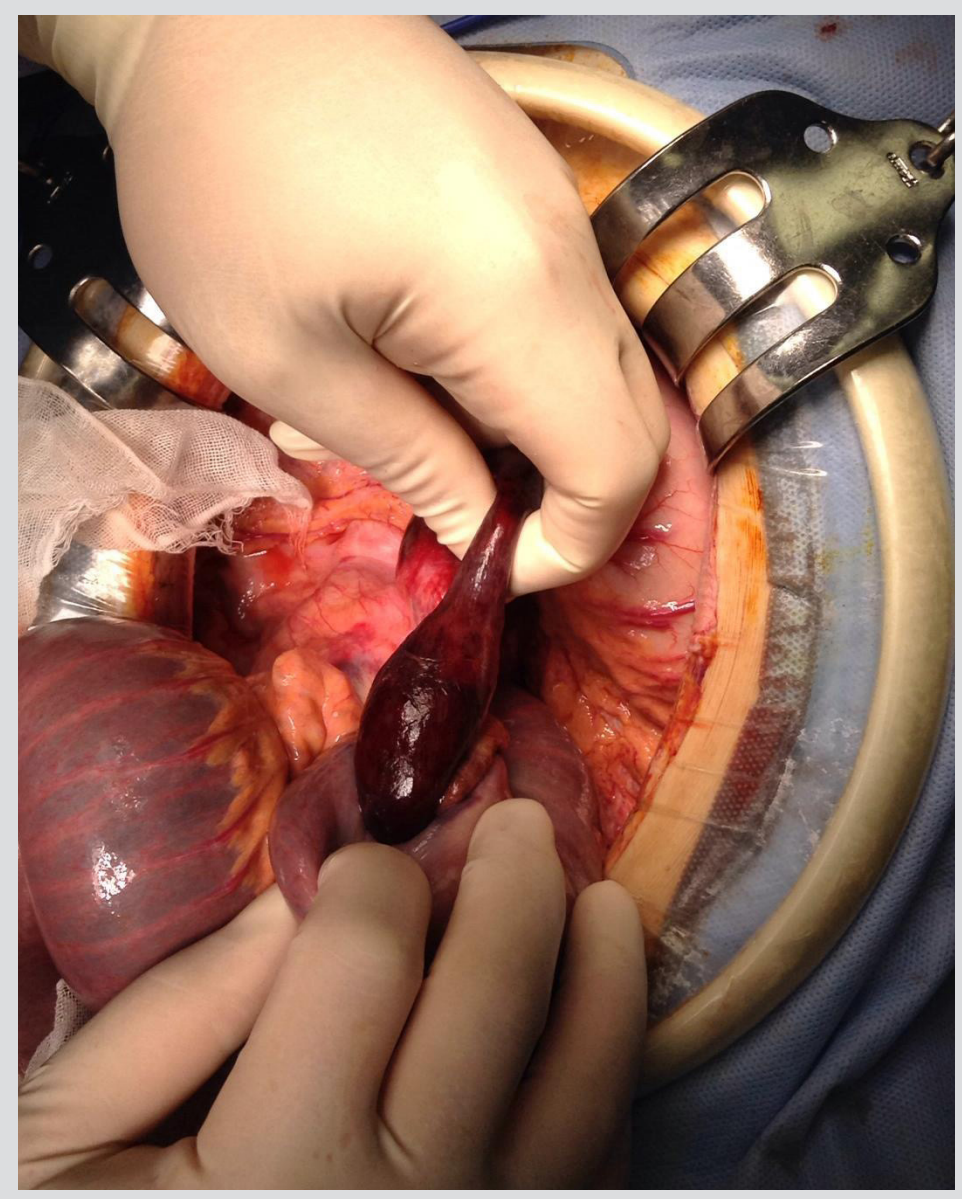

Figure 2. Antegrade jejunal intussusception with ischaemic change 


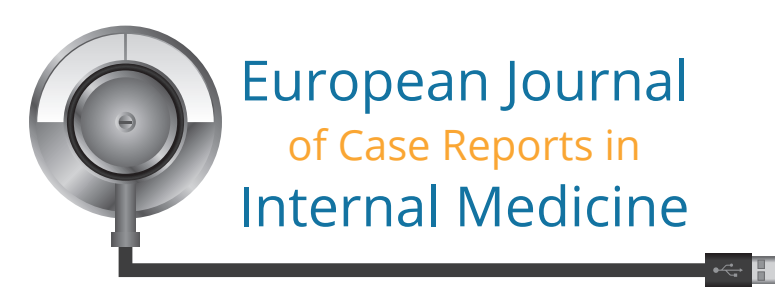

\section{DISCUSSION}

Intussusception is a rare but fatal complication caused by placement of a nasointestinal ileus tube in the small intestine ${ }^{[1]}$. Most intussusceptions associated with a nasointestinal ileus tube are antegrade ${ }^{[1]}$. As in our case, this complication often occurs a few days after placement, and an abdominal mass can be palpated at the site of obstruction on physical examination ${ }^{[2]}$. Because urgent operative reduction is needed to avoid intestinal resection in most cases, early diagnosis and surgical reduction of intussusception are critical ${ }^{[3]}$. However, the diagnosis of intussusception associated with a nasointestinal ileus tube is often delayed because many physicians are unaware of this complication due to its rarity. Therefore, a diagnosis of intussusception should be suspected if abdominal pain and distention worsen after placement of a nasointestinal ileus tube ${ }^{[1]}$.

\section{REFERENCES}

1. Peskin SR, Langevin Jr RE, Banks PA. Proximal jejunal intussusception associated with a long tube. Dig Dis Sci 1986;31(6):657-60.

2. Poppel MH. Ileal intussusception as result of intestinal intubation. JAMA 1959;169(11):1189-90.

3. Redmond P, Ambos M, Berliner L, Pachter HL, Megibow A. latrogenic intussusception: a complication of long intestinal tubes. Am J Gastroenterol 1982;72(1):39-42. 